\title{
Coronary Artery Responsiveness to Ergonovine Provocation in Patients Without Vasospatic Angina
}

\section{A Quantitative Coronary Angiography Analysis}

\author{
Kyeong Ho Yun, ${ }^{1}$ MD, Seoung-Nam Shin, ${ }^{1}$ MD, Jum Suk Ko, ${ }^{1}$ MD, Sang Jae RHeE, ${ }^{1}$ MD, \\ Nam-Ho KIm, ${ }^{1}$ MD, Seok Kyu OH, ${ }^{1} \mathrm{MD}$, and Jin-Won JEONG, ${ }^{1}$ MD
}

\begin{abstract}
SUMmary
Even patients without vasospastic angina show vasoconstriction after intracoronary ergonovine administration. We evaluated the determinants of coronary artery responsiveness to ergonovine in such patients.

In 165 patients with no provoked electrocardiographic changes or ischemic chest pain during an intracoronary ergonovine test, total cholesterol, triglycerides (TG), high density lipoprotein cholesterol (HDL), and low density lipoprotein cholesterol (LDL) were correlated with the arterial luminal diameters before and after ergonovine infusion and after nitroglycerin injection by quantitative coronary angiography analysis.

The mean and maximal basal tone (ie, percent change between baseline luminal diameter and diameter after nitroglycerin) were $7.0 \pm 9.9 \%$ and $27.9 \pm 10.8 \%$, respectively. The mean and maximal responsiveness to ergonovine (ie, percent change between minimal diameter during ergonovine infusion and diameter after nitroglycerin) were $30.3 \pm 13.6 \%$ and $52.7 \pm 16.0 \%$, respectively. The TG level $(r=0.191, P=0.016)$ and TG/HDL ratio $(r=0.182, P=0.021)$ were positively correlated with the basal tone, whereas LDL level $(r=0.155, P=0.048)$ and LDL/HDL ratio $(r=0.172, P=$ 0.030 ) were positively correlated with the responsiveness to ergonovine. By multivariate analysis, LDL level, LDL/HDL ratio, and smoking were independent predictors of more than $50 \%$ responsiveness to ergonovine.

Serum lipid profile and smoking influence the basal tone and responsiveness to ergonovine of coronary artery in patients without vaospastic angina. (Int Heart J 2011; 52: 338-342)
\end{abstract}

Key words: Ergonovine, Coronary vasospasm, Lipids

$\mathrm{E}$ gonovine provocation test is used to detect coronary artery spasm in patients with suspected variant angina. The predictive accuracy of this test in patients with recurrent angina at rest and electrocardiographic changes is excellent, but even patients with atypical symptoms demonstrate a moderate nonspecific vasoconstrictive response. ${ }^{3)}$ In the presence of intact vascular endothelium, ergonovine induces early and transient vasodilation followed by sustained dose-dependent vasoconstriction. However, endothelial dysfunction reduces nitric oxide activity, resulting in pronounced and sustained vasoconstriction after ergonovine administration. ${ }^{4,5)}$

Considering these findings, the coronary artery response to ergonovine may be associated with coronary risk factors causing endothelial dysfunction even in patients without vasospastic angina. The aim of the present study was to evaluate the determinants of coronary artery responsiveness to ergonovine provocation in patients without vasospastic angina by quantitative coronary angiography (QCA) analysis.

\begin{abstract}
METHODS
Study population: We retrospectively analyzed 165 patients with chest pain and nonsignificant coronary stenosis $(<50 \%$ diameter stenosis). Each patient was referred for definitive evaluation after presenting with atypical chest pain, defined by uncommon or no precipitating events, inconsistent location, unusual duration of pain, or inconclusive response to nitroglycerin. The exclusion criteria were cases of positive results in noninvasive tests and those of coronary intervention, previous myocardial infarction, and congestive heart failure, or documented cardiomyopathy. The study protocol was approved by the Institutional Review Board of the hospital.

After overnight fasting, blood sampling was done to measure total cholesterol, triglyceride (TG), high density lipoprotein cholesterol (HDL), low density lipoprotein cholesterol (LDL), lipoprotein (a), and high-sensitivity C-reactive protein (hsCRP) levels. Complete blood count (CBC) with a differential count was measured within 24 hours before coronary angiography.

Ergonovine provocation test and angiographic analysis: All drug medications that could influence the coronary artery response to ergonovine were stopped 48 hours before testing, ex-
\end{abstract}

From the ${ }^{1}$ Department of Cardiovascular Medicine, Regional Cardiocerebrovascular Center, Wonkwang University Hospital, Iksan, Republic of Korea.

This study was supported by grants from Wonkwang University in 2010.

Address for correspondence: Kyeong Ho Yun, MD, Department of Cardiovascular Medicine, Regional Cardiocerebrovascular Center, Wonkwang University Hospital, 344-2 Shinyong-dong, Iksan, Jeonbuk 570-711, Korea.

Received for publication April 13, 2011.

Revised and accepted July 8, 2011. 
cept short-acting nitrate. In patients in which significant coronary stenosis was absent, the ergonovine provocation test was performed, as previously reported. ${ }^{6,7)}$ Ergonovine was injected at $10 \mu \mathrm{g} /$ minute for 4 minutes for a maximal dose of $40 \mu \mathrm{g}$ into the right coronary artery and at $16 \mu \mathrm{g} /$ minute over 4 minutes for a total dose of $64 \mu \mathrm{g}$ into the left coronary artery, with at least a 5 minute interval between each injection; and then, the occurrence of chest pain, the change in ST segment on eletrocardiography (ECG), and the development of spasm on coronary angiography were observed. After the test, coronary angiograms were again followed by intracoronary injection of nitroglycerin $(200 \mu \mathrm{g})$. We excluded the patients with positive results, defined as cases in which more than $99 \%$ of focal vasospasm was noted on coronary angiography in the presence of typical chest pain or ischemic ECG changes.

Coronary arteriography images were digitalized and analyzed with a QCA imaging system (CAAS 5.0, Pie Medical Imaging, Maastricht, The Netherlands) by an observer blinded to the patient's clinical data (Figure 1). The reference coronary artery diameter and minimal luminal diameter (MLD) were measured in all American Heart Association (AHA) segments except the diagonal branch and obtuse marginal branch (only the first branch was measured). The basal arterial tone was defined as the percent change between the baseline MLD and the diameter after nitroglycerin injection.

Basal tone $(\%)=$

MLD after nitroglycerin - Baseline MLD $\times 100$ MDL after nitroglycerin

Coronary artery responsiveness to ergonovine was defined as the percent change between the MLD during ergonovine infusion and the diameter after nitroglycerin injection. Coronary responsiveness to ergonovine $(\%)=$

$\underline{\text { MLD after nitroglycerin - MDL after ergonovine }} \times 100$ MDL after ergonovine

Small coronary arteries $(<1 \mathrm{~mm}$ diameter after nitroglycerin) or segments overlapped by other branches were not analyzed.

Statistical analyses: All measurements are represented as the mean \pm standard deviation. Continuous variables were com-

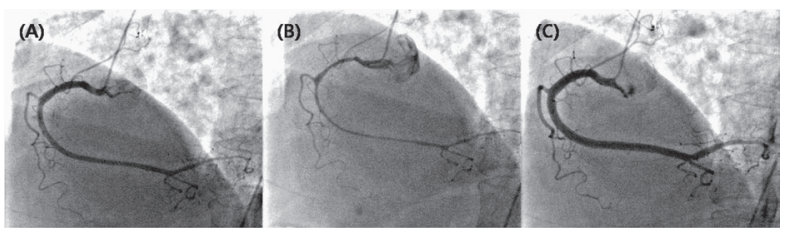

Figure 1. Representative result of the quantitative coronary angiography analysis. We performed arteriography at baseline (A), after ergonovine infusion (B), and after nitroglycerin injection (C). For each coronary artery segment, the minimal luminal diameter (MLD) was measured and the basal tone and responsiveness to ergonovine were calculated. The MLDs of the proximal, mid, and distal segments in this case were as follows; 2.89, 2.18 , and $2.13 \mathrm{~mm}$ at baseline, $1.66,1.50$, and $0.67 \mathrm{~mm}$ after ergonovine infusion, and 3.80, 3.64, and $3.49 \mathrm{~mm}$ after nitroglycerin injection, respectively. Therefore, the basal tone of the proximal, mid, and distal segments was $23.95,40.11$, and $38.97 \%$, respectively; the maximal basal tone was $40.11 \%$ and the average value was $34.34 \%$. The responsiveness of the proximal, mid, and distal segments to ergonovine was 56.32, 58.79, and $80.80 \%$; the maximal responsiveness was $80.80 \%$, and the average responsiveness was $65.04 \%$. pared by $t$-tests, using SPSS 12.0 for Windows (SPSS Inc., Chicago, IL). Correlations between the serum lipids and the ergonovine induced changes in coronary artery diameter were analyzed using Pearson's correlation coefficients. To perform multivariate analysis, we adopted a $50 \%$ vasoconstrictive response to ergonovine on the basis of the median value. Statistical significance was set at $P<0.05$ (two-tailed).

\section{RESUltS}

All patients underwent the full provocation protocol, and only mild side effects not requiring premature termination of the test were observed. Seven patients experienced atypical chest discomfort, but no significant ST-segment abnormalities were observed. Normal sinus rhythm was maintained in each patient, without precipitation of heart block or significant arrhythmia. The clinical characteristics of the patients and the QCA results are presented in Table I. The mean of maximal baseline diameter stenosis of each patient by QCA was $16.9 \pm$ $8.1 \%$. The mean basal tone of all coronary artery segments was $7.0 \pm 9.9 \%$, and the average of the maximal value of each patient was $27.9 \pm 10.8 \%$. Mean coronary responsiveness to ergonovine was $30.3 \pm 13.6 \%$, and the average of the maximal value of each patient was $52.7 \pm 16.0 \%$. One hundred two patients exhibited more than $50 \%$ of maximal responsiveness to ergonovine $(61.8 \%)$.

The mean lipid values were $190.4 \pm 41.9,154.7 \pm 107.3$, $50.4 \pm 32.5$, and $122.7 \pm 35.4 \mathrm{mg} / \mathrm{dL}$ for total cholesterol, TG, HDL, and LDL, respectively. The TG level and TG/HDL ratio were positively correlated with the baseline diameter stenosis (TG: $r=0.206, P=0.009$; TG/HDL: $r=0.174, P=0.028$ ) and basal tone (TG: $r=0.191, P=0.016$; TG/HDL: $r=0.182$, $P=0.021$ ) (Figure 2A, 2B). On the other hand, the LDL level and LDL/HDL ratio were significantly correlated with coronary responsiveness to ergonovine (LDL: $r=0.155, P=$ 0.048; LDL/HDL: $r=0.172, P=0.030$ ) (Figure 2C). In the patients with more than $50 \%$ of maximal responsiveness to ergonovine, LDL level and LDL/HDL ratio were more closely correlated with coronary responsiveness to ergonovine (LDL: $r=0.232, P=0.021$; LDL/HDL: $r=0.219, P=0.031$ ) (Figure 3 ). There was no correlation between the lipoprotein (a) and hsCRP levels and the basal tone or coronary responsiveness to ergonovine.

Table I. Baseline Clinical and QCA Characteristics

\begin{tabular}{lc}
\hline Patients $(n)$ & 165 \\
Age (years) & $56.8 \pm 11.2$ \\
Male (\%) & $45(27.3)$ \\
Hypertension (\%) & $62(37.6)$ \\
Diabetes (\%) & $22(13.3)$ \\
Current smoker (\%) & $22(13.3)$ \\
Maximal baseline diameter stenosis $(\%)$ & $16.9 \pm 8.1$ \\
Maximal diameter stenosis after ergonovine infusion $(\%)$ & $24.2 \pm 17.7$ \\
Maximal diameter stenosis after nitroglycerin injection $(\%)$ & $17.2 \pm 8.1$ \\
Maximal basal coronary arterial tone $(\%)$ & $27.9 \pm 10.8$ \\
Maximal responsiveness to ergonovine $(\%)$ & $52.7 \pm 16.0$
\end{tabular}

Values are expressed as $n(\%)$ or mean \pm standard deviation. QCA indicates quantitative coronary angiography. 
A

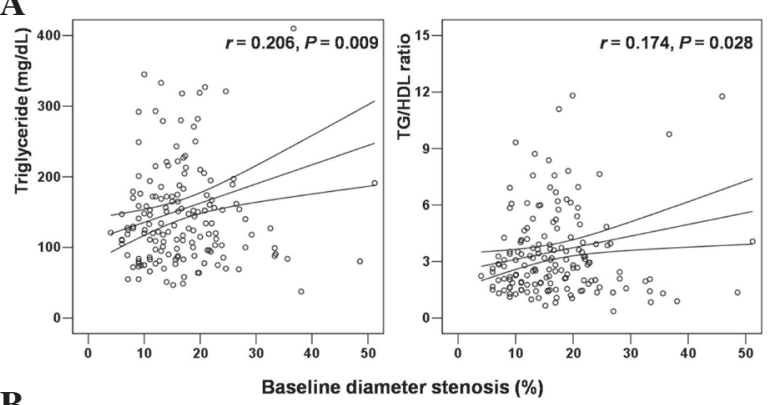

B

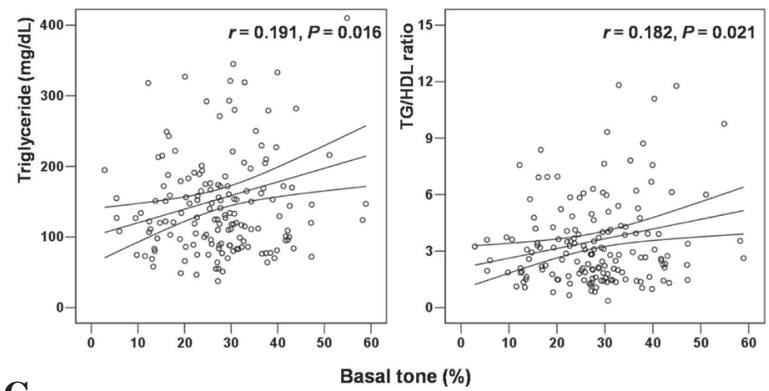

C

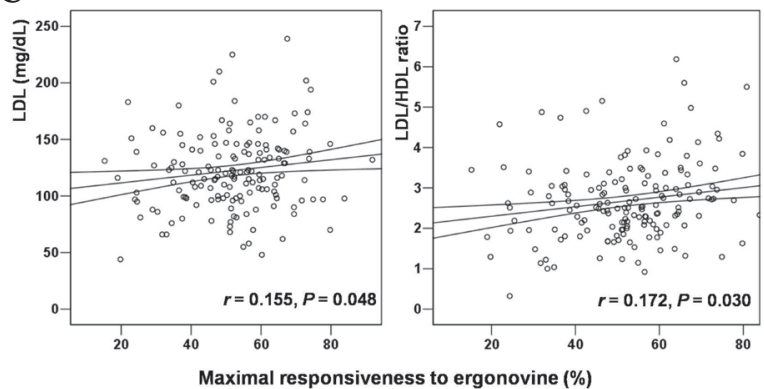

Figure 2. A: Correlations of the baseline diameter stenosis with the triglyceride (TG) level and ratio of TG and high density lipoprotein cholesterol (HDL) level. B: Correlations of the basal tone with the TG level and TG/HDL ratio. C: Correlations of the maximal responsiveness to ergonovine with low density lipoprotein cholesterol (LDL) level and LDL/HDL ratio.

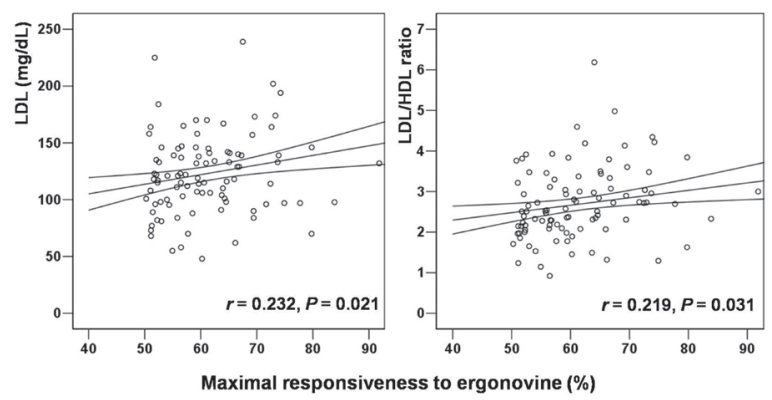

Figure 3. Correlations of the maximal responsiveness to ergonovine with low density lipoprotein cholesterol (LDL) level and LDL/high density lipoprotein cholesterol (HDL) ratio in patients with more than $50 \%$ of maximal responsiveness to ergonovine.

To evaluate the effects of other risk factors on coronary responsiveness to ergonovine, we analyzed the data according to the presence or absence of each risk factor. There were no

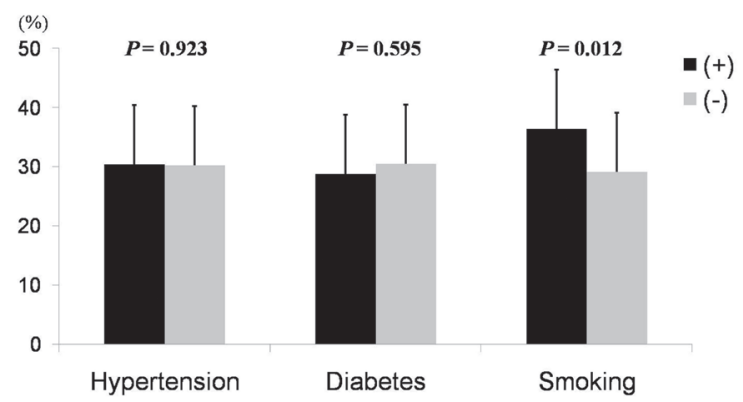

Figure 4. Coronary artery responsiveness to ergonovine in the presence $(+)$ and absence (-) of hypertension, diabetes, and smoking. The data are the mean \pm standard deviation.

Table II. Multivariate Analysis for the Prediction of More Than 50\% of Maximal Responsiveness to Ergonovine

\begin{tabular}{lccc}
\hline & OR & $95 \%$ CI & $P$ \\
\hline LDL level & 1.07 & $1.02-1.13$ & 0.007 \\
LDL/HDL ratio & 1.20 & $1.01-1.68$ & 0.022 \\
Smoking & 4.14 & $1.17-14.7$ & 0.028 \\
WBC count & 1.00 & $1.00-1.00$ & 0.108 \\
HDL level & 0.93 & $0.84-1.02$ & 0.121 \\
Total cholesterol level & 0.98 & $0.96-1.01$ & 0.161 \\
TG/HDL ratio & 1.49 & $0.71-3.14$ & 0.288 \\
Age $>$ 65 years & 1.35 & $0.60-3.00$ & 0.471 \\
Triglyceride & 0.99 & $0.98-1.01$ & 0.494 \\
hsCRP level & 0.99 & $0.96-1.03$ & 0.581 \\
\hline
\end{tabular}

LDL indicates low density lipoprotein cholesterol; WBC, white blood cells; HDL, high density lipoprotein cholesterol; TG, triglycerides; and hsCRP, high-sensitivity C-reactive protein.

significant differences in the basal tone between the patients with hypertension, diabetes, or smoking and those without risk factors. The coronary responsiveness to ergonovine was not influenced significantly by gender, presence of hypertension, or presence of diabetes. However, the smokers had a pronounced vasoconstrictive response to ergonovine compared to the nonsmokers $(36.4 \pm 13.6 \%$ versus $29.1 \pm 13.4 \%, P=0.012)(\mathrm{Fig}-$ ure 4). Multivariate analysis revealed that LDL level $(\mathrm{OR}=$ $1.07, P=0.007)$, LDL/HDL ratio $(\mathrm{O} R=1.20, P=0.022)$, and smoking $(\mathrm{O} R=4.14, P=0.028)$ were independent predictors of more than $50 \%$ of maximal responsiveness to ergonovine (Table II).

\section{Discussion}

In this study, we have demonstrated that smoking and serum lipids could influence the basal tone and coronary responsiveness to ergonovine. In patients without significant stenosis and vasospastic angina, the TG level and TG/HDL ratio were correlated with the basal tone, and the LDL level and LDL/ HDL ratio were correlated with the coronary responsiveness to ergonovine.

Coronary artery spasm plays an important role in the pathogenesis of various ischemic heart diseases, including not only variant angina but also unstable angina, myocardial in- 
farction and sudden death. ${ }^{8)}$ Heupler, et al introduced the ergonovine provocation study for the diagnosis of coronary vasospasm. ${ }^{1)}$ Ergonovine provokes coronary vasospasm through a complex mechanism involving serotonin receptors, direct stimulation of $\alpha$ receptors, and inhibition of nitric oxide (NO) release from the endothelium. ${ }^{9,10)}$ A certain degree of nonspecific vasoconstriction can be observed in all individuals receiving ergonovine. Kimball, et al demonstrated a progressive, dose-related reduction in coronary dimensions that was not related to the induction of chest discomfort or ECG changes. ${ }^{11)}$ The clinician should not confuse this nonspecific response with true vasospastic angina. However, there is little information available about coronary "hyper-responsiveness" to ergonovine. $^{12,13)}$

The mechanism of coronary vasospasm includes hypersensitivity to endothelium derived factors, platelet derived vasoactive substances, and autonomic nervous tone. ${ }^{14,15)}$ The basal coronary tone is related to the regulation of vascular smooth muscle tension by local endothelium derived factors, humoral factors, and parasympathetic nervous tone. ${ }^{16,17)}$ Thus, similar factors may play a role in both vasospasm and the basal coronary tone, and vascular endothelial dysfunction in particular can be a critical factor. Endothelial dysfunction leads to reduced NO activity, resulting in more pronounced and sustained vasoconstriction after ergonovine administration. ${ }^{4,5)}$ Smoking reduces NO activity via oxygen radicals in cigarette smoke. ${ }^{18)}$ Therefore, unsurprisingly, smoking is the only established risk factor for vasospasm. ${ }^{1920)}$ In our study, coronary responsiveness to ergonovine was the most accentuated in smokers, but not in patients with hypertension or diabetes.

Animal studies showed a relationship between experimental atherosclerosis and vasoconstriction. ${ }^{21-23)}$ However, clinical studies revealed controversial results. Nobuyoshi, et al and Harding, et al did not find any relationship between coronary vasospasm and total cholesterol and LDL levels. ${ }^{20,24)}$ On the other hand, Kugiyama, et al have shown that oxidized LDL levels are significantly and positively correlated with the vasoconstrictive response of coronary arteries. ${ }^{25)}$ Nedelijkovic, et al reported that total cholesterol and LDL levels are significantly correlated with the baseline MLD and coronary vasoconstriction induced by ergonovine. ${ }^{26)}$ We found a significant but weak relationship between the LDL level and the coronary responsiveness to ergonovine. The interstudy differences may be attributable to differences in the study populations; many studies of the relationship between the lipid profile and vasospasm included some patients with true vasospastic angina. However, we demonstrated that the LDL level may play some role in the coronary responsiveness to ergonovine even in patients without variant angina who have nonspecific vasoconstriction.

The TG level is also an important factor for atherosclerosis and endothelial dysfunction. In a hypertriglyceridemic state, the accumulation of remnants results in a proinflammatory and oxidative milieu that may enhance the expression of adhesion molecules, foam cell formation, and smooth muscle cell toxicity. ${ }^{27)}$ High TG levels induce an increase in the small dense LDL level, which most easily causes atherosclerosis; and also the reduction of HDL, thereby accelerating atherosclerosis. ${ }^{28)}$ However, there are few reports on the relationship between the TG level and variant angina so far. We demonstrated that the TG level, especially the TG/HDL ratio, is significantly related to the basal tone. Basal coronary artery tone is increased in patients with variant angina in proportion to the disease activity. ${ }^{29,30)}$ Therefore, our data suggest that the LDL level is related to the development of vasospastic angina, and the TG level is related to the disease activity of vasospastic angina.

This study has several limitations. We did not clarify the clinical significance of the basal tone and responsiveness to ergonovine in patients with chest pain syndrome and "almostnormal" coronary arteries. This study should be considered as a suggestion regarding how to identify coronary risk factors affecting the pathogenesis of coronary vasospasm. Coronary angiography frequently underestimates the atherosclerotic plaque burden in positively remodeled coronary sites; therefore, the effects of LDL and HDL levels on MLD could be underestimated in this study.

In conclusion, in patients with atypical chest pain and nonspecific vasoconstriction to ergonovine, smoking and serum lipids influence the coronary basal tone and responsiveness to ergonovine. Although the clinical significance of the coronary artery response to ergonovine in these patients has not been definitively elucidated, the clinician must control the risk factors, because they are closely related to the occurrence of coronary artery disease as well as variant angina.

\section{REFERENCES}

1. Heupler FA Jr, Proudfit WL, Razavi M, et al. Ergonovine maleate provocative test for coronary arterial spasm. Am J Cardiol 1978; 41: 631-40.

2. Cipriano PR, Guthaner DF, Orlick AE, Ricci DR, Wexler L, Silverman JF. The effects of ergonovine maleate on coronary arterial size. Circulation 1979; 59: 82-9.

3. Curry RC Jr, Pepine CJ, Sabom MB, Feldman RL, Christie LG, Conti CR. Effects of ergonovine in patients with and without coronary artery disease. Circulation 1977; 56: 803-9.

4. Hori T, Matsubara T, Ishibashi T, et al. Decrease of nitric oxide end-products during coronary circulation reflects elevated basal coronary artery tone in patients with vasospastic angina. Jpn Heart J 2000; 41: 583-95.

5. Kugiyama $\mathrm{K}$, Yasue $\mathrm{H}$, Okumura $\mathrm{K}$, et al. Nitric oxide activity is deficient in spasm arteries of patients with coronary spastic angina. Circulation 1996; 94: 266-71.

6. Igarashi Y, Yamazoe M, Shibata A. Effect of direct intracoronary administration of methylergonovine in patients with and without variant angina. Am Heart J 1991; 121: 1094-100.

7. Sueda S, Kohno H, Fukuda H, et al. Clinical impact of selective spasm provocation tests: comparisons between acetylcholine and ergonovine in 1508 examinations. Coron Artery Dis 2004; 15: 491-7.

8. Hillis LD, Braunwald E. Coronary-artery spasm. N Engl J Med 1978; 299: 695-702. (Review)

9. Angus JA, Cocks TM, Satoh K. The alpha adrenoceptors on endothelial cells. Fed Proc 1986; 45: 2355-9.

10. Karila-Cohen D, Delpy E, Dubois-Randé JL, et al. Influence of the endothelium, nitric oxide and serotonergic receptors on coronary vasomotor responses evoked by ergonovine in conscious dogs. Br J Pharmacol 1999; 127: 1039-47.

11. Kimball BP, LiPreti V, Aldridge HE. Quantitative arteriographic responses to ergonovine provocation in subjects with atypical chest pain. Am J Cardiol 1989; 64: 778-82.

12. Sunagawa O, Shinzato Y, Touma T, Tomori M, Fukiyama K. Differences between coronary hyperresponsiveness to ergonovine and vasospastic angina. Jpn Heart J 2000; 41: 257-68.

13. Kashima K, Tachibana H, Nakamura K, Kano T, Tanaka Y, Arima $\mathrm{T}$. Long-term outcome of patients with ergonovine induced coro- 
nary constriction not associated with ischemic electrocardiographic changes. J Cardiol 2001; 37: 301-8.

14. Vanhoutte PM, Shimokawa H. Endothelium-derived relaxing factor and coronary vasospasm. Circulation 1989; 80: 1-9. (Review)

15. Kim NH, Jeong JW, Park EM, et al. Alterations of autonomic nervous activity associated with spontaneous coronary spasm in patients with variant angina. Korean Circ J 2004; 34: 362-7.

16. Bassenge E. Flow-dependent regulation of coronary vasomotor tone. Eur Heart J 1989; 10: 22-7. (Review)

17. Ozaki Y, Keane D, Serruys PW. Relation of basal coronary tone and vasospastic activity in patients with variant angina. Heart 1996; 75: 267-73.

18. Kugiyama K, Yasue H, Ohgushi M, et al. Deficiency in nitric oxide bioactivity in epicardial coronary arteries of cigarette smokers. J Am Coll Cardiol 1996; 28: 1161-7.

19. Sugiishi M, Takatsu F. Cigarette smoking is a major risk factor for coronary spasm. Circulation 1993; 87: 76-9.

20. Nobuyoshi M, Abe M, Nosaka H, et al. Statistical analysis of clinical risk factors for coronary artery spasm: identification of the most important determinant. Am Heart J 1992; 124: 32-8.

21. Kawachi Y, Tomoike H, Maruoka Y, et al. Selective hypercontraction caused by ergonovine in the canine coronary artery under conditions of induced atherosclerosis. Circulation 1984; 69: 44150 .

22. Dalessandri KM, Lantz BM, Tsukamoto H, Link DP, Brock J. Superior mesenteric artery vasoactivity in hyperlipidemic Watanabe rabbits versus normal lipidemic New Zealand controls. J Invest
Surg 1989; 2: 471-7.

23. Heistad DD, Armstrong ML, Marcus ML, Piegors DJ, Mark AL. Augmented responses to vasoconstrictor stimuli in hypercholesterolemic and atherosclerotic monkeys. Circ Res 1984; 54: 711-8.

24. Harding MB, Leithe ME, Mark DB, et al. Ergonovine maleate testing during cardiac catheterization: a 10 -year perspective in 3,447 patients without significant coronary artery disease or Prinzmetal's variant angina. J Am Coll Cardiol 1992; 20: 107-11.

25. Kugiyama K, Sugiyama S, Soejima H, et al. Increase in plasma levels of oxidized low-density lipoproteins in patients with coronary spastic angina. Atherosclerosis 2001; 154: 463-7.

26. Nedeljkovic MA, Ostojic MC, Beleslin BD, et al. Ergonovine-induced changes of coronary artery diameter in patients with nonsignificant coronary artery stenosis : relation with lipid profile. Herz 2007; 32: 329-35.

27. Yu KC, Cooper AD. Postprandial lipoproteins and atherosclerosis. Front Biosci 2001; 6: D332-54. (Review)

28. Kolovou GD, Anagnostopoulou KK, Daskalopoulou SS, Mikhailidis DP, Cokkinos DV. Clinical relevance of postprandial lipaemia. Curr Med Chem 2005; 12: 1931-45. (Review)

29. Lee SJ, Park SJ, Park SW, et al. Increased basal tone and hyperresponsiveness to acetylcholine and ergonovine in spasm-related coronary arteries in patients with variant angina. Int J Cardiol 1996; 55: 117-26.

30. Ozaki Y, Keane D, Serruys PW. Relation of basal coronary tone and vasospastic activity in patients with variant angina. Heart 1996; 75: 267-73. 